\title{
Biologia populacional de Emerita brasiliensis Schmitt (Crustacea, Hippidae) na Praia Vermelha do Norte, Ubatuba (São Paulo, Brasil)
}

\author{
Jelly Makoto Nakagaki ${ }^{1,3}$ \\ Marcelo Antonio Amaro Pinheiro 2,3
}

\begin{abstract}
Population biology of Emerita brasiliensis Schmitt (Crustacea, Hippidae) in Vermelha do Norte beach, Ubatuba (São Paulo, Brazil). Emerita brasiliensis Schmitt, 1935 is a common mole crab called "tatuira" in Brazil, with distribution from Espírito-Santo State (Brazil) to Buenos Aires Province (Argentina). Specimens were collected bimonthly from May/1992 through March/1993 with 4mm siffers mesh, in the intertidal region at Vermelha do Norte Beach, Ubatuba, São Paulo, Brazil. The animals were sexed and measured with a precision caliper of $0.1 \mathrm{~mm}(\mathrm{CL}$ $=$ carapace length). Males presented CL amplitude from 3.4-17.3 $\mathrm{mm}(13.2 \pm 2.1 \mathrm{~mm})$, and females from $13.8-26.3 \mathrm{~mm}(20.5 \pm 1.8 \mathrm{~mm})$, showing a well-defined sexual dimorfism for this variable. The population presented a smaller incidence of males in relation to females (0.54:1.00), however in May/1992 an inverse pattern occurred (1.84:1.00). Ovigerous females were present in all samples with exception of May and September/1992 with greater frequencies in July/1992 and January/1993. It is probable that female maturity occurs is reached with $17 \mathrm{~mm} \mathrm{CL}$, the smallest size in wich ovigerous females were found.
\end{abstract}

KEY WORDS. Hippidae, Emerita brasiliensis, population structure, sex-ratio

Os hipídeos são decápodos que habitam águas tropicais e temperadas em todos os oceanos, estando distribuídos desde a zona costeira até áreas de maior profundidade. Segundo Tereza Cristina dos Santos Calado (comunicação pessoal), Hippidae Latreille, 1825 é representada no Brasil por três espécies, sendo duas do gênero Emerita Scopoli, 1777 - E. brasiliensis Schmitt, 1935 e E. portoricensis Schmitt, 1935 - e apenas uma do gênero Hippa Fabricius, 1787 - H. testudinaria (Herbst, 1791).

Emerita brasiliensis é um anomuro muito abundante na zona intertidal de praias arenosas, encontrado-se distribuído no Atlântico Ocidental, com registros para o México, Venezuela, Trinidad, Brasil (ES ao RS) e Uruguai (EFFORD 1976). Estas espécies ocupam ambientes onde estão sujeitas a grande estresse em função da variação térmica, ação de ondas, correntes, declividade da praia e predação. Segundo Bowman \& Dolan (1985), as populações de E. talpoida (Say, 1817) são fortemente influenciadas pelas características físicas da praia, como a granulometria da areia e a energia da arrebentação da maré, fazendo com que apresente uma migração peculiar em função destes estímulos.

1) Unidade de Jardim, Universidade Estadual de Mato Grosso do Sul. Avenida 11 de dezembro, 79240-000 Jardim, Mato Grosso do Sul, Brasil.

2) Departamento de Biologia Aplicada, FCAVJ, Universidade Estadual Paulista. 14870-000 Jaboticabal, São Paulo, Brasil.

3) Núcleo de Estudos em Biologia, Ecologia e Cultivo de Crustáceos (NEBECC). 
As espécies do gênero Emerita têm se destacado na literatura carcinológica por sua estrutura populacional complexa, podendo auxiliar na elucidação de padrões reprodutivos, muitas vezes desconhecidos. Vários fenômenos reprodutivos peculiares já foram verificados neste grupo, entre os quais destacam-se a neotenia (EFFORD 1967; SUBRAMONIAM 1977), reversão sexual (BARNES \& WENNER 1968; WENNER \& HALEY 1981) e hermafroditismo protândrico (SUBRAMONIAM 1981).

A estrutura populacional dos crustáceos têm sido analisada, principalmente, pela distribuição dos exemplares em classes de tamanho, razão sexual, dinâmica temporal, crescimento e taxas de natalidade/mortalidade. Tais informações são de grande importância para o melhor entendimento da biologia de uma espécie, particularmente no que se refere a $E$. brasiliensis, que tem sido pouco abordada na literatura (VELOSO \& CALAZANS 1992; Veloso \& VALENTIN 1993).

O objetivo do presente trabalho é avaliar a proporção sexual e distribuição dos exemplares de E. brasiliensis em classes de tamanho, utilizando amostras de uma população da Praia Vermelha do Norte, Ubatuba, São Paulo.

\section{MATERIAL E MÉTODOS}

A Praia Vermelha do Norte localiza-se na Enseada de Ubatuba, Município

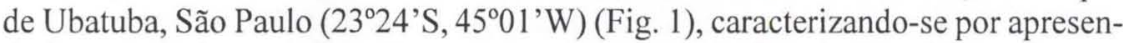
tar intenso batimento de ondas, perfil vertical com declive de 10 a $16^{\circ}$ e substrato arenoso composto, principalmente, por areia de granulação média $(0,25-0,5 \mathrm{~mm})$ e grossa $(0,5-1,0 \mathrm{~mm})$.

Coletas bimensais de E. brasiliensis foram realizadas durante a maré baixa, no período matutino, de maio/1992 a março/1993, abrangendo a zona de lavado da região intertidal. A área foi percorrida por uma extensão de $500 \mathrm{~m}$ no sentido norte e sul para detecção de agrupamentos de indivíduos da espécie, onde foram realizados dois transectos, em paralelo e perpendicularmente ao mar. Em cada transecto foram realizados cinco pontos de amostragem a cada $50 \mathrm{~cm}$, utilizando um tubo amostrador de PVC com $15 \mathrm{~cm}$ de diâmetro, para retirada de uma coluna de sedimento de $30 \mathrm{~cm}$. Os exemplares obtidos foram separados do sedimento arenoso com auxílio de peneiras com $4 \mathrm{~mm}$ de malha e mantidos em sacos plásticos devidamente etiquetados. Após realizada a identificação dos hipídeos, os exemplares da espécie foram sexados, classificados em grupos de interesse (machos, fêmeas sem ovos e fềmeas ovígeras) e mensurados da extremidade do rostro até a margem posterior do cefalotórax $(\mathrm{CC}=$ comprimento cefalotorácico $)$, utilizando um paquímetro de precisão $0,1 \mathrm{~mm}$ (Fig. 2).

Para verificar a estrutura populacional da espécie, os exemplares foram distribuídos em classes de tamanho $(1 \mathrm{~mm})$, determinando-se a freqüência de cada grupo de interesse em relação ao total de indivíduos amostrados durante o período de estudo. O mesmo foi realizado para cada coleta bimensal, visando determinar o recrutamento populacional.

A proporção sexual (macho:fêmea) foi calculada para o total de indivíduos, para cada coleta bimensal e nas classes de tamanho. Neste último caso, foi confeccionado um gráfico de pontos relativo ao percentual de machos nas classes de tamanho, seguindo o método descrito por WENNER (1972), cuja curva resultante foi comparada com os padrões descritos por esse autor. 


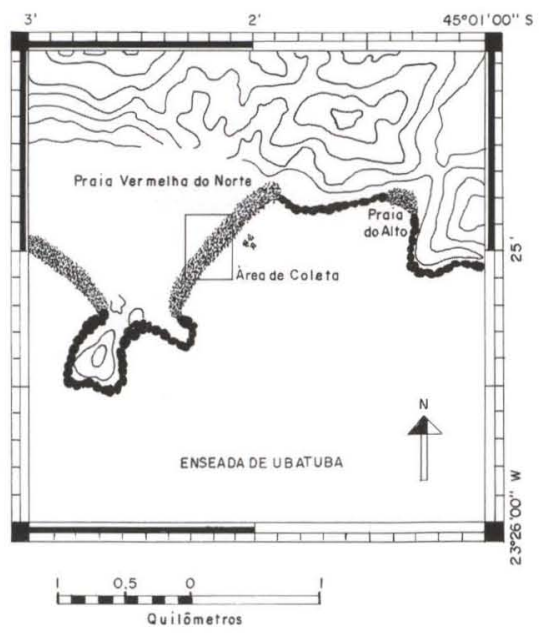

Fig. 1. Localização geográfica da Praia Vermelha do Norte, Ubatuba, São Paulo, Brasil.

O teste "t" de Student foi empregado para verificar a existência de uma possível diferença de tamanho (CC) entre os sexos, para o total de indivíduos amostrados. O teste do $X^{2}$ foi utilizado para verificar se a proporção sexual obtida em cada coleta diferiu de 1:1. Em todos os casos o nível de significância estatística foi estabelecido em $5 \%$.

\section{RESULTADOS}

Durante o período de estudo foram coletados 834 indivíduos de E. brasiliensis, representados por 290 machos e 544 fêmeas (97 ovígeras e 447 sem ovos).

A amplitude de variação do comprimento da carapaça dos machos foi de $13,9 \mathrm{~mm}(3,4 \mathrm{a} 17,3 \mathrm{~mm})$, pouco maior em relação à das fêmeas que foi de $12,5 \mathrm{~mm}$ (13,8 a 26,3mm). As médias de CC obtidas para cada sexo apresentaram valores contrastantes entre si, sendo de $13,2 \pm 2,1 \mathrm{~mm}$ para os machos e $20,5 \pm 1,8 \mathrm{~mm}$ para as fêmeas $(t=-53,24 ; \mathrm{p}<0,05)$, evidenciando uma bimodalidade na distribuição de freqüência em tamanho (Fig. 3). As primeiras fêmeas ovígeras na população ocorreram a partir de $17 \mathrm{~mm}$.

Analisando-se a distribuição de freqüência dos exemplares nos meses de amostragem (Fig. 4), percebe-se um deslocamento modal relativamente pequeno ao longo do tempo, com recrutamento dos machos verificado no período do verão.

De modo geral os machos ocorreram em menor número na população estudada (1,00: 1,87), com significância estatística apenas nos meses de setembro e novembro/1992. No mês de maio/1992 este quadro se inverteu, mostrando um maior número de machos do que fêmeas $(1,84: 1,00)$ (Fig. 5, Tab. I).

Excetuando-se os meses de maio e setembro/1992, as fêmeas ovígeras ocorreram nas demais coletas bimensais, apresentando maior freqüência em julho/1992 (inverno) e uma segunda incidência de menor freqüência em janeiro e março/1993 (verão) (Fig. 5). 


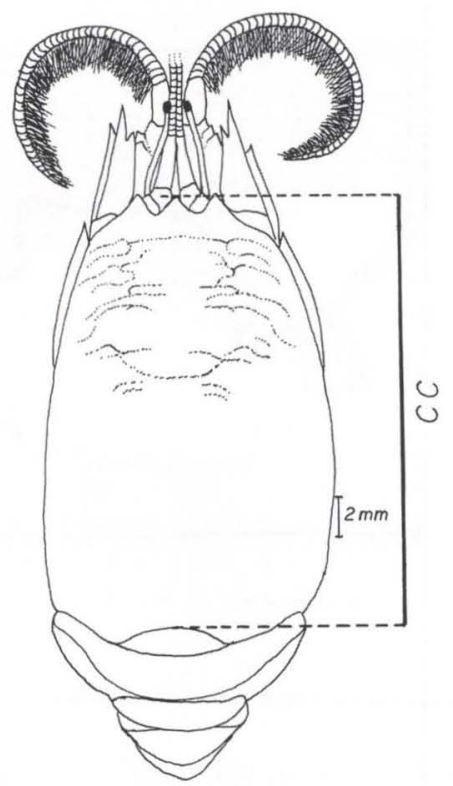

Fig. 2. Emerita brasiliensis. Vista dorsal de um exemplar adulto. (CC) Comprimento da carapaça.

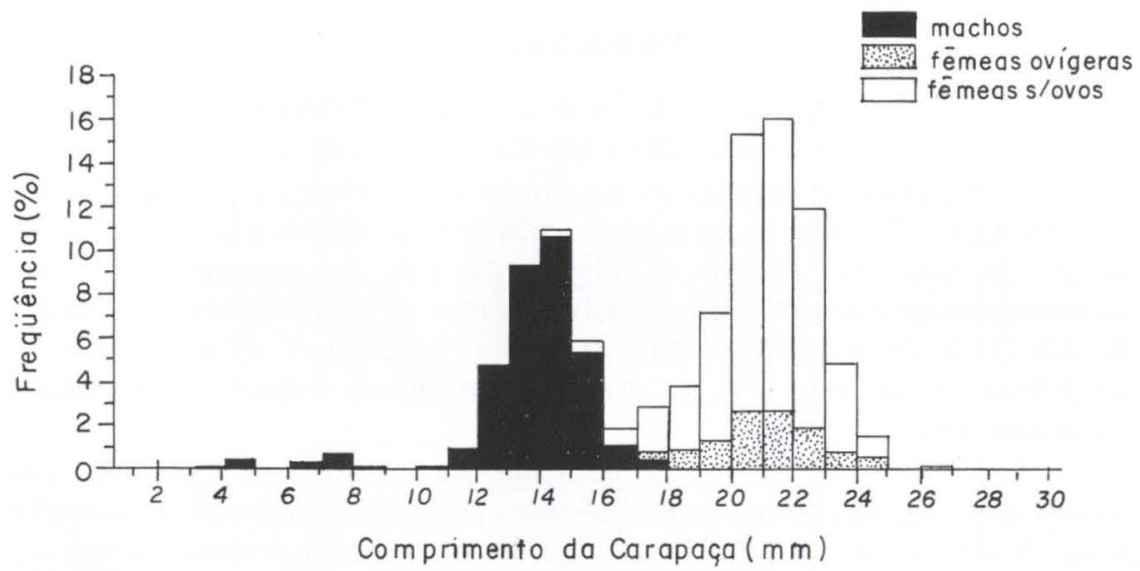

Fig. 3. Emerita brasiliensis. Distribuição de freqüência dos grupos de interesse (machos, fêmeas não ovigeras e fêmeas ovigeras) nas classes de tamanho.

Na figura 6 pode-se perceber a presença exclusiva de machos nas classes de tamanho iniciais, decréscimo nas classes intermediárias $(15<\mathrm{CC}<20 \mathrm{~mm})$ e completa ausência nas classes finais. A partir de $17,3 \mathrm{~mm}$ não foi verificada a presença de machos na população. 


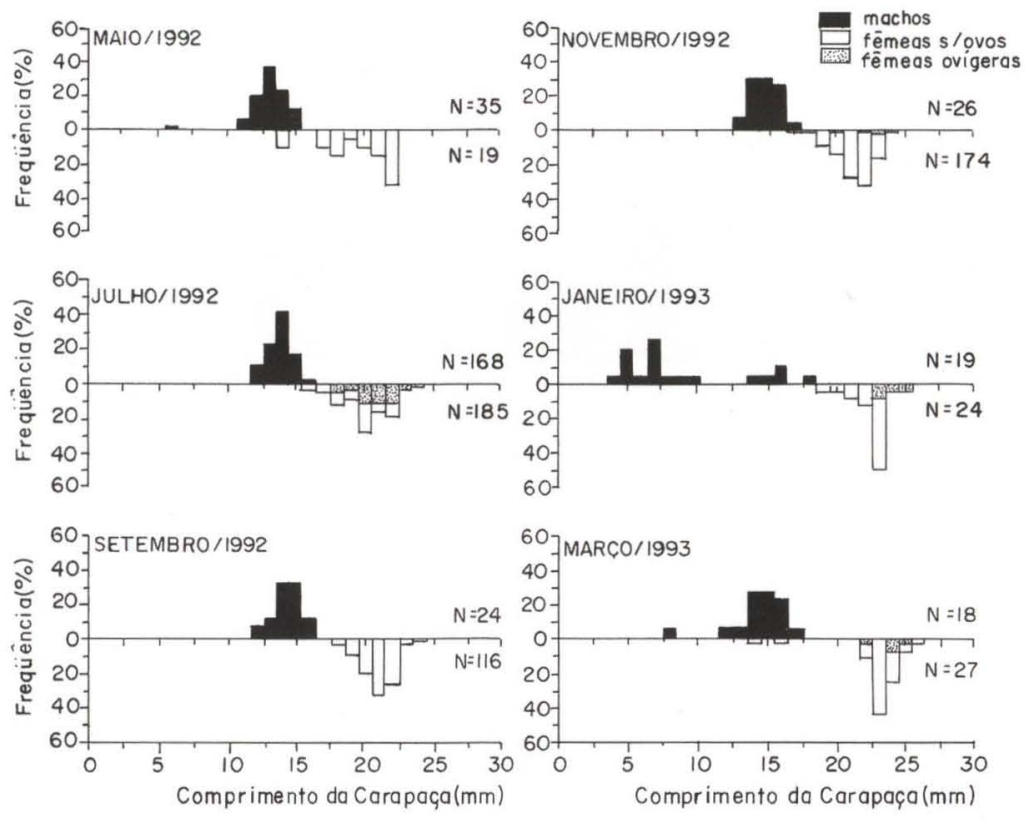

Fig. 4. Emerita brasiliensis. Distribuição de freqüência dos grupos de interesse (machos, fêmeas não ovígeras e fêmeas ovígeras) por classes de tamanho em cada um dos meses de coleta durante o período de estudos.

Tabela I. Proporção sexual de E. brasiliensis em cada coleta bimensal durante o período de maio/1992 a março/1993, na Praia Vermelha do Norte, Ubatuba, São Paulo.

\begin{tabular}{lcccccc}
\hline & Maio/92 & Julho/92 & Setembro/92 & Novembro/92 & Janeiro/93 & Março/93 \\
\hline Macho: fêmea & $1,84: 1,00$ & $1,00: 1,09$ & $1,00: 4,83$ & $1,00: 6,69$ & $1,00: 1,26$ & $1,00: 1,50$ \\
$X^{2}$ & $4,74^{*}$ & $0,73 \mathrm{~ns}$ & $60,46^{*}$ & $109,52^{*}$ & $0,58 \mathrm{~ns}$ & $1,80 \mathrm{~ns}$ \\
\hline
\end{tabular}

(*) $p<0,05$; (ns) $p>0,05$.

\section{DISCUSSÃO}

O crescimento somático diferencial entre os sexos é muito freqüente nas espécies do gênero Emerita, cujos machos atingem geralmente pequeno porte e maturidade sexual precoce (EFFORD 1967; PENCHASZADEH 1971; SUBRAMONIAM 1977; SASTRE 1991). O mesmo foi verificado para Emerita brasiliensis, cujos machos apresentaram média de comprimento da carapaça cerca de $64 \%$ do tamanho médio das fêmeas, valor próximo aos $50 \%$ encontrados para $E$. analoga por MACGINITIE (1938). De acordo com EFFORD (1967) poucas espécies de hipídeos apresentam machos com tamanho similar ao das fêmeas, entre as quais figuram Emerita austroafricanus Schmitt, 1937 e E. holthuisi Sankolli, 1965. Este fenômeno pode ser explicado por taxas de crescimento ou mortalidade diferenciais entre os sexos (EFFORD 1967; ANSELL et al. 1972) ou evidência de reversão sexual (WENNER 1972; WENNER \& HALEY 1981). 


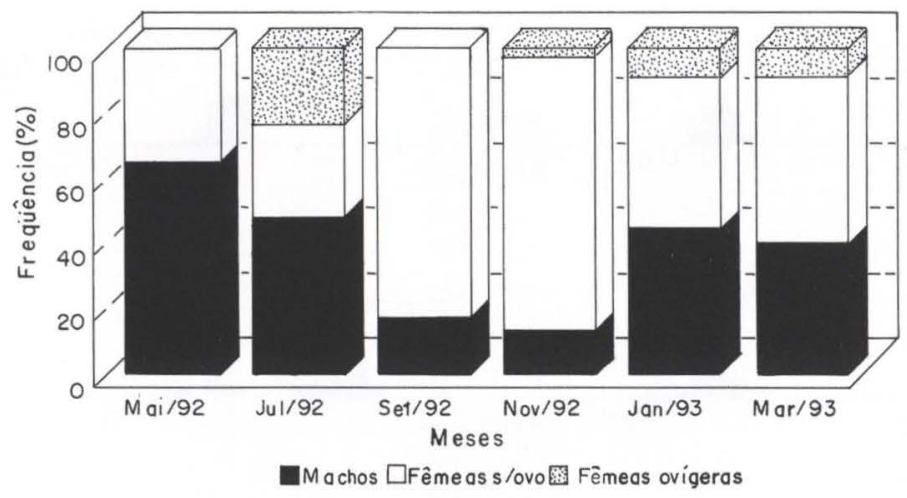

Fig. 5. Emerita brasiliensis. Freqüência dos grupos de interesse (machos, fêmeas não ovigeras e fêmeas ovígeras) nos meses de coleta durante o período de estudos.

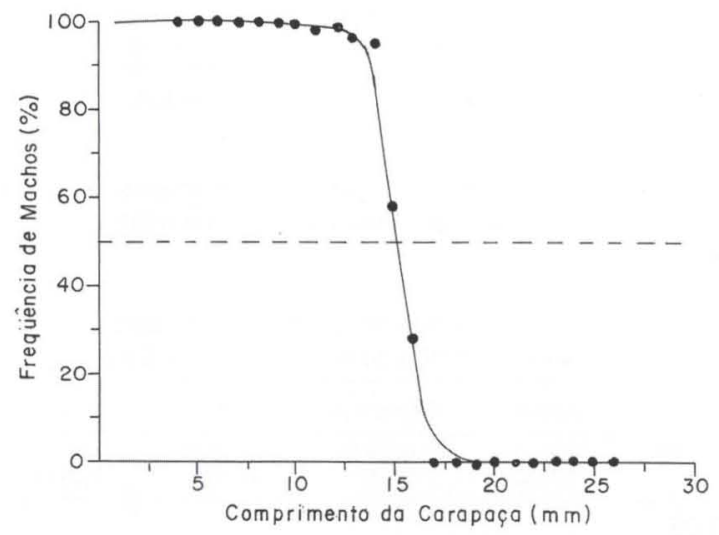

Fig. 6. Emerita brasiliensis. Percentual de machos em relação ao percentual de fêmeas nas classes de tamanho. (CC) Comprimento da carapaça.

Com base nos resultados obtidos para a proporção sexual em função do tamanho, a explicação mais plausível para a completa ausência de fêmeas de $E$. brasiliensis com $\mathrm{CC}<17,3 \mathrm{~mm}$ seria a reversão sexual, levando a classificá-la no padrão reverso descrito por WENNER (1972). Neste sentido, padrões distintos também já foram registrados para outros hipídeos, como é o caso de E. analoga (Stimpson, 1857) e Hippa pacifica (Dana, 1852), classificadas no padrão anômalo e intermediário, respectivamente. Os experimentos de laboratório conduzidos com E. analoga revelam reversão sexual em cerca de $21 \%$ dos machos, o que é maximizado com a elevação da temperatura ambiente. Até o momento tais informações não são disponíveis para $E$. brasiliensis. 
Durante a cópula da maioria das espécies de Emerita, é muito comum que dois ou mais machos copulem a mesma fêmea, à qual são encontrados geralmente aderidos (EFFORD 1967). Embora tenha-se registrado uma maior proporção macho: fêmea de E. brasiliensis apenas na coleta de maio/1992, os dados obtidos no presente estudo impedem inferências sobre a época de cópula dessa espécie, o que possivelmente poderia ser constatado numa amostragem mensal da população.

Os resultados obtidos por VELOSO \& VALENTIN (1993) revelam uma maior abundância larval de E. brasiliensis nos meses de verão, decréscimo durante o outono/primavera e ausência no inverno. Tal periodicidade reprodutiva foi similar à descrita para E. analoga por MACGINITIE (1938) e BARNES \& WENNER (1968), diferindo da constatada no presente estudo, onde um pulso reprodutivo foi verificado no inverno, seguido por outro de menor intensidade nos meses de primavera/verão. Esta periodicidade diferencial pode estar relacionada com características físicas peculiares ao local de estudo, haja visto que alterações das correntes oceânicas, ciclos de maré e movimentação da areia ao longo da praia também afetam o comportamento gregário e a distribuição destes animais (BARNES \& WENNER 1968). Segundo BOWMAN \& DOLAN (1985) ondas de grande energia e/ou aumento da maré podem promover um declínio populacional causado pela migração dos animais para a região infralitoral. HALEY (1982) verificou tal fato para as fêmeas maturas de Hippa pacifica, que ocupam primariamente a metade inferior da área de lavagem, onde existe uma maior facilidade na captura de alimento, causada pela maior energia das ondas. Apesar desses padrões migratórios, o presente trabalho revelou as maiores taxas de captura para o período de inverno, coincidindo com a principal época reprodutiva da espécie.

A observação de fêmeas ovígeras de E. brasiliensis com CC $>17 \mathrm{~mm}$ corrobora a afirmação de EFFORD (1967), que estabelece o início da maturidade das fêmeas do gênero Emerita acima de $12 \mathrm{~mm}$. Segundo o mesmo autor, é comum que os machos deste gênero estejam maturos tão logo chegam às praias, ainda como megalopas.

O acompanhamento das principais modas de distribuição de E. brasiliensis, a cada dois meses sucessivos, permite inferir que essa espécie apresenta uma taxa de crescimento lenta, confirmada pelo registro de estágios juvenis a partir da quinta classe de tamanho ( $\mathrm{CC}>4 \mathrm{~mm}$ ) no mês de janeiro/93 (verão), provavelmente resultantes da desova de julho/92 (inverno). Segundo EFFORD (1970) o desenvolvimento larval de E. analoga ocorre em aproximadamente 4 meses, enquanto GIANUCA (com. pess.) estimou uma variação de 2 a 3 meses para E. brasiliensis no sul do Brasil. Apesar da longevidade das larvas ser determinada geneticamente para cada espécie, fatores extrínsecos como a qualidade e disponibilidade de alimento, salinidade e, principalmente, a temperatura, podem influenciar o tempo de permanência das larvas no plâncton (PINHEIRO et al. 1994).

Apesar de E. brasiliensis ser uma espécie abundante e com ampla distribuição na região intertidal, poucos estudos enfocam sua biologia. Entre outras abordagens, a confirmação de reversão sexual em experimentos de laboratório viria a facilitar a caracterização da dinâmica populacional desta espécie, favorecendo uma maior compreensão do crescimento e longevidade de cada sexo. 


\section{REFERÊNCIAS BIBLIOGRÁFICAS}

Ansell, A.D.; P. Sivadas; B. Narayanan \& A. Trevallion. 1972. The ecology of two sandy beaches in South West India. II. Notes on Emerita holthuisi. Mar. Biol. 17: 311-317.

BARNES, N.B. \& A.M. WENNER. 1968. Seasonal variation in the sand crab Emerita analoga (Decapoda, Hippidae) in the Santa Barbara area of California. Limnol. Oceanog. 13: 465-475.

BOWMAN, M.L. \& R. DOLAN. 1985. The relationship of Emerita talpoida to beach characteristics. Jour. Coastal Res. 1 (2): 151-163.

EFForD, I.E. 1967. Neoteny in sand crabs of the genus Emerita (Anomura, Hippidae). Crustaceana 13: 81-93.

-1970. Recruitment to sedentary marine populations as exemplified by the sand crab, Emerita analoga (Decapoda, Hippidae). Crustaceana 18: 293-308.

- 1976. Distribution of the sand crabs in the genus Emerita (Decapoda, Hippidae). Crustaceana 30 (2): 169-183.

HALEY, S.R. 1982. Zonation by size of the pacific mole crab, Hippa pacifica Dana (Crustacea: Anomura: Hippidae), in Hawaii. Jour. Exp. Mar. Biol. Ecol. 58: 221-231.

MacGinitie, G.E. 1938. Movements and mating habits of the sand crab, Emerita analoga. Amer. Midl. Nat. 19: 471-481.

PENCHASZADEH, P.E. 1971. Observaciones cuantitativas preliminares en playas arenosas de la Costa Central del Peru con especial referencia a las poblaciones de muy-muy (Emerita analoga) (Crustacea, Anomura, Hippidae). Inst. Biol. Mar. (Mar del Plata) Contrib. 177: 1-16.

PINHEIRO, M.A.A.; A. FrANSOZO \& M.L. NEGREIROS-Fransozo. 1994. Estimativa da duração larval em função da temperatura para a Família Majidae (Crustacea, Decapoda, Brachyura). Bol. Inst. Pesca, São Paulo, 21: 75-81.

SAstre, M.P. 1991. Sex specific growth and survival in the mole crab Emerita potoricensis (Schmitt). Jour. Crust. Biol. 11 (1): 103-112.

Subramoniam, T. 1977. Aspects of sexual biology of the anomuran crab Emerita asiatica. Mar. Biol. 43: 369-377.

- 1981. Protandric hermaphroditism in a mole crab, Emerita asiatica (Decapoda, Anomura). Biol. Bull. 160 (1): 161-174.

Veloso, V.G. \& D.K. Calazans. 1992. Descrição dos estágios larvais de Emerita brasiliensis Schmitt, 1935 (Decapoda: Hippidae) através de plancton, Rio Grande - RS. Nerítica 7: 133-145.

Veloso, V.G. \& J.L. VALENTIN. 1993. Larval distribution and seasonal abundance of Emerita brasiliensis Schmitt, 1935 (Decapoda, Hippidae) in southern Brazil. Rev. Brasil. Biol. 53 (1): 131-141.

WENNER, A.M. 1972. Sex ratio as a function of size in marine crustacea. Amer. Natur. 106: 321-350.

WenNER, A.M. \& S.R. HALEY. 1981. On the question of sex reversal in mole crabs (Crustacea, Hippidae). Jour. Crust. Biol. 1 (4): 506-517. 